\title{
High tumor cell vimentin expression indicates prolonged survival in patients with ovarian malignant tumors
}

\author{
Sebastian Szubert ${ }^{1}$, Krzysztof Koper ${ }^{1}$, \\ Magdalena Maria Dutsch-Wicherek ${ }^{2}$, Wojciech Jozwicki ${ }^{3}$ \\ ${ }^{1}$ Chair of Oncology, Radiotherapy and Gynecologic-Oncology of the Ludwik Rydygier Medical College in Bydgoszcz, \\ Nicolaus Copernicus University, Torun, Poland \\ ${ }^{2}$ Student of the Faculty of Medicine, Jagiellonian University Medical College, Cracow, Poland \\ ${ }^{3}$ Department of Tumour Pathology and Pathomorphology, Ludwik Rydygier Collegium Medicum in Bydgoszcz, \\ Nicolaus Copernicus University in Torun, Torun, Poland
}

\begin{abstract}
Objectives: The main aims of the study were to investigate the expression of vimentin and its correlation with the overall survival (OS) of patients with malignant ovarian tumors, and the correlation between vimentin expression and tumor stroma characteristics.

Material and methods: The study focused on 94 malignant ovarian tumors that had been collected from women who were treated in the Department of Gynecology and Oncology of the Lukaszczyk Oncological Center, Bydgoszcz, Poland. Vimentin expression was assessed in both the cancer cells (expression intensity and quantitative analysis) and the tumor stroma (expression intensity). Vimentin expression was analyzed according to both stromal cellularity and the clinicopathological features of the disease.

Results: Both high cancer cell vimentin expression intensity and high quantitative vimentin expression (up to and including $30 \%$ of cells) indicated a significantly prolonged OS. Low vimentin stromal expression was associated with prolonged OS, although the difference did not reach the level of significance. High tumor cell vimentin expression intensity was associated with significantly higher vimentin stromal expression. High vimentin expression in the tumor stroma indicated a significantly higher cellularity of the tumor stroma. Vimentin expression in cancer cells and the tumor stroma were not dependent on the histopathological type, the tumor grade or the FIGO stageof the disease.

Conclusions: High cancer cell vimentin expression is associated with an improved OS of patients with malignant ovarian tumors. The expression of vimentin in ovarian malignancies may influence the structure of the tumor stroma.
\end{abstract}

Key words: vimentin; ovarian neoplasms; tumor microenvironment

Ginekologia Polska 2019; 90, 1:11-19

\section{INTRODUCTION}

Epithelial ovarian cancer (EOC) is the leading cause of death from gynecological malignancies as well as the fifth leading cause of cancer-related death among women in the Western countries. A woman's lifetime risk of developing EOC is $1.3 \%$, meaning that one in 78 women will be affected by EOC. Although complete remission following primary treatment is achieved in approximately one half of all patients, the majority of these patients will relapse, and the disease will then become fatal [1,2].
Multiple factors influence the prognosisfor EOC patients. Patient overall survival (OS) is affected by the degree of residual disease following surgery, the histological type of the tumor, the type of first-line chemotherapy, the stage of the disease, and the age of the patient at diagnosis [3]. Until recently, cancer studies were mainly focused on the characteristics of the cancer cells themselves. However, it is now known that some features of the tumor stromacan impact patient OS. For example, in a study by Chen et al., patients with stroma-rich ovarian malignancies had 
a worse prognosis and a higher risk of relapse compared with patients with tumors with poorly developed stroma [4]. Multiple stromal cells, such as cancer-associated fibroblast (CAFs), tumor-infiltrated lymphocytes (TILs), and tumor-associated macrophages (TAMs) secrete growth factors and immunomodulating cytokines that facilitate cancer progression. The development of new blood vessels enables tumor growth and may influence the cancer cell phenotype. The role of tumor stroma is especially important when it comes to cancer invasion and metastasis [5].Cancer cells can communicate with stromal cells and modify stromal architecture and cell content, creating a more permissive microenvironment to facilitate its own growth [6]. Angiogenesis is one of the most important stromal targets for ovarian cancer therapy. Anti-vascular-endothelial growth factor (VEGF) therapy has a well-established position in the primary treatment of non-optimally debulked ovarian cancer [7]. However, novel therapies targeting stromal cells, such as CAFs and TILs, are also being developed and applied [8-10].

Vimentin, a $57 \mathrm{kDa}$ protein, is one of the cytoskeletal proteins belonging to the type III intermediate filament protein family. Vimentin expression in adults is found in a variety of organs and in multiple normal cells [11]. However, vimentin expression increases during cancerogenesis [12]. In addition, vimentin is expressed in numerous cells of the tumor stroma, thus its expression in tumor stroma may reflect the conditions within the tumor microenvironment [13-15]. Vimentin was traditionally considered a marker of epithelial-to-mesenchymal transition (EMT), the process whereby cells lose their epithelial characteristics and acquirea spindle-shape and the characteristics of mesenchymal cells. EMT is a characteristic phenomenon of cancer cells; associated with tumor infiltrative growth, metastasis and greater resistance to chemotherapy [16]. However, vimentin is not only a cytoskeletal protein responsible for cell architecture and integrity, but also a participant in the regulation of cancer-related signal transduction pathways, such as Raf/14-3-3 and PI3K/AKT, as well asin the extracellular signal-regulated kinase (Erk) pathways responsible for invasiveness, motility, and cell migration. Vimentin is a crucial element of cell migration, invasion, invadopodia development and metastases, and these processes are inhibited by vimentin downregulation [17]. Additionally, current data suggest the important function of vimentin in VEGF-driven angiogenesis, indicating the role of vimentin in tumor stroma rebuilding [18] its exploitation as a clinical target has achieved limited success and novel molecular targets are required.

High expression of vimentin in cancer cells is associated with a poor prognosis for patients with numerous types of neoplasms. Recent studies have also emphasized that elevated stroma vimentin expression correlates with shortened survival rates in colorectal cancer patients[19, 20].
Although the role of vimentin as a prognostic factor in ovarian cancer has not yet been established, preclinical studies have provided evidence that elevated vimentin expression is associated with platinum sensitivity which suggests a favorable prognosis [21, 22].

\section{Objectives}

The main aim of our research was to evaluate the impact of vimentin expression in ovarian cancer cells and in tumor stromaon the overall survival of patients with malignant ovarian tumors. Furthermore, we tried to determine whether vimentin expression correlates with selected elements of tumor stroma and pathologic characteristics of cancer.

\section{MATERIAL AND METHODS}

\section{Patient characteristics}

The study material comprised 94 samples of malignant ovarian tumors collected from a group of women treated in the Department of Gynecology and Oncology of the Lukaszczyk Oncological Center, Bydgoszcz, Poland, during the period from 2005 to 2010. The median patient age was 57 (range 25-86). The materials were obtained during primary cytoreductive surgery. The distribution of histopathological findings and FIGO stage of the disease are summarized in Table 1.The median patient follow-up was 41 months (range 0-160 months). Information on patients who died was retrieved from the

\begin{tabular}{|l|l|}
\hline \multicolumn{2}{|l}{ Table 1. Distribution of histopathological findings and FIGO stage } \\
of the disease among patients included in the study \\
\hline Histopathological diagnoses & Number [\%] \\
\hline Serous adenocarcinoma & $57(61 \%)$ \\
\hline Mucinous adenocarcinoma & $5(5 \%)$ \\
\hline Endometrioid adenocarcinoma & $11(12 \%)$ \\
\hline Clear cell adenocarcinoma & $4(4 \%)$ \\
\hline Undifferentiated carcinomas & $2(2 \%)$ \\
\hline Transitional cell carcinomas & $3(3 \%)$ \\
\hline Adult granulosa cell tumors & $10(11 \%)$ \\
\hline Borderline ovarian tumors & $2(2 \%)$ \\
\hline Tumor grading system* & Number [\%] \\
\hline G1 & $2(2 \%)$ \\
\hline G2 & $30(36 \%)$ \\
\hline G3 & $52(62 \%)$ \\
\hline FIGO stage of the disease & Number [\%] \\
\hline FIGO I & $20(21 \%)$ \\
\hline FIGO II & $8(9 \%)$ \\
\hline FIGO III & $66(70 \%)$ \\
\hline FIGO IV** & 0 \\
\hline &
\end{tabular}

*Histopathological grading system was not applied for the granulosa cell tumors; **Patients with the disease classified as stage IV were not included in the study 
database of the Cuiavia-Pomerania regional office of the National Health System of Poland. This study received the approval of the Jagiellonian University Ethics Committee (Reference: KBET/89/B/2005 and DK/KB/CM/0031/447/2010), and all patients gave informed written consent.

\section{Immunohistochemistry}

The study was performed on a DakoLink48 apparatus, using the EnVision method, with EnVision ${ }^{\mathrm{TM}}$ Flex+ kit, Mouse, High pH (Link) (Dako/Agilent, K8002) and ready-to-use murine monoclonal antibody from Dako/Agilent Vimentin (IR630), clone V9. All immunohistochemical studies were performed on $4 \mu \mathrm{m}$ thick sections taken from cancerous tumors fixed in $4 \%$ formalin buffered and embedded in paraffin blocks. Paraffin sections were placed on Knittel Glass adhesive slides and incubated for 2 hours in a chamber thermostat at $60^{\circ} \mathrm{C}$. Prior to the automatic performance of the study, tissue sections were subjected to dewaxing followed by thermal epitope detection (HIER) in a PTLink device using EnVision ${ }^{\mathrm{TM}}$ FLEXTarget Retrieval Solution, High pH (50x) (K8002). Finally, the preparations were dehydrated in a series of alcohols with increasing concentrations and closed in a medium (Consul Mount, Thermo Shandon, USA). The method takes place at room temperature. Each time, a control preparation was added to the series of patient samples. Vimentin immunoreactivity was assessed within the cancer cells and the tumor stroma.The differentiation between stromal and cancer cells infiltrating the tumor stroma was assessed based on cell morphology, and the assessment was performed by an experienced pathologist (W.J.). In the case of cancer cells we used two types of immunoreactivity assessment: 1) evaluation of the staining intensity, and 2) evaluation of the percentage of positive cells. Staining intensity in vimentin cancer cells was stratified into two groups: 1) low expression, including absent $(0)$, minimal $(1+)$ and moderate $(2+)$ expression; and 2) high expression (3+). Vimentin quantitative expression in cancer cells was evaluated using the subjective method of the succeeding approximations assessing the percentage of vimentin positive cells. For our statistical analysis, we distinguished two study groups: 1) comprised of low quantitative vimentin expression (staining found in less than $30 \%$ of cells), and 2) comprised of high quantitative vimentin expression (vimentinexpression presentin morethan $30 \%$ of cells).Vimentin expression in tumor stroma was assessed by staining intensity. Staining intensity was evaluated by subjective assessment in central and peripheral stroma using a four-degree scale: absent (0), minimal (1+), moderate (2+) and high (3+). The results from the central and peripheral stroma were averaged, and then the expression in the tumor stroma was assessed as either "low" when the results of the calculations were below and including 2, or "high"when above 2. Stromal cellularity was evaluated by assessing the number of tumor stroma cells visible in the microscopic High Power Field (HPF) view. The quantity of tu- mor stroma cells was evaluated subjectively in the central and peripheral stroma using a three-degree system: $1+, 2+$ and $3+$, and then the results from the central and peripheral stroma were averaged. Subsequently, the cellularity was assessed as either "low" when the results of calculations were below 1.5, or "high" when above and including 1.5. Immunohistochemistry studies and tumor analyses were performed by an experienced pathologist (W.J.), who was blinded for the results of patients' overall survival. Representative images of vimentin staining are presented in Figure 1.

\section{Statistical analysis}

The data were compared using achi square test. However, if expected cell frequencies were below fiveforthe $2 \times 2$ contingency table, then the Fisher exact test was performed. Survival analyses were conducted using Kaplan-Meier survival curves.

\section{RESULTS}

\section{Vimentin expression and patient OS}

Table 2 summarizes the results of the analysis of vimentin expressionand stromal cellularity.

High cancer cell vimentin expression intensity was associated with significantly prolonged survival. Patients whose cancer cells had high vimentin expression intensity had a median OS of 100 months (range 0-160) compared with 32 months (range 0-160) for the patients with low vimentin expression intensity in cancer cells $(P=0.007)$ (Fig. $2 A)$.

Patients who suffered from tumors with high quantitative vimentin expression in the cancer cells (up to and including $30 \%$ of cells) had a significantly longer median OS (49 months, range 0-160) compared with those patients with low quantitative vimentin expression in the cancer cells (below $30 \%$ of cells; 28 months, range $3-134 ; \mathrm{P}=0.02$ ) (Fig. 2B).

Patients with low vimentin expression in the tumor stroma hada prolonged OS when compared with patients with high expression. However, thatresult did not achieve a level of statistical significance ( 117 months, range $0-160$ vs 37 months, range $0-148$ respectively, $P=0.06$; Fig. $1 C$ ). The cellularity of the tumor stroma had no impact on patients' $O S$.

\section{Relationship between tumor and stroma vimentin expression, and the correlation with stroma cellularity}

High tumor cell vimentin expression intensity was associated with significantly higher vimentin stromal expression. There wasno relationship between vimentin quantitative expression in cancer cells and vimentin expression in the tumor stroma (Tab. 3). High vimentin expression in the tumor stroma was correlated with high cellularity of the tumor stroma. Vimentin expression in cancer cells was not associated with tumor stroma cellularity (Tab. 4). 

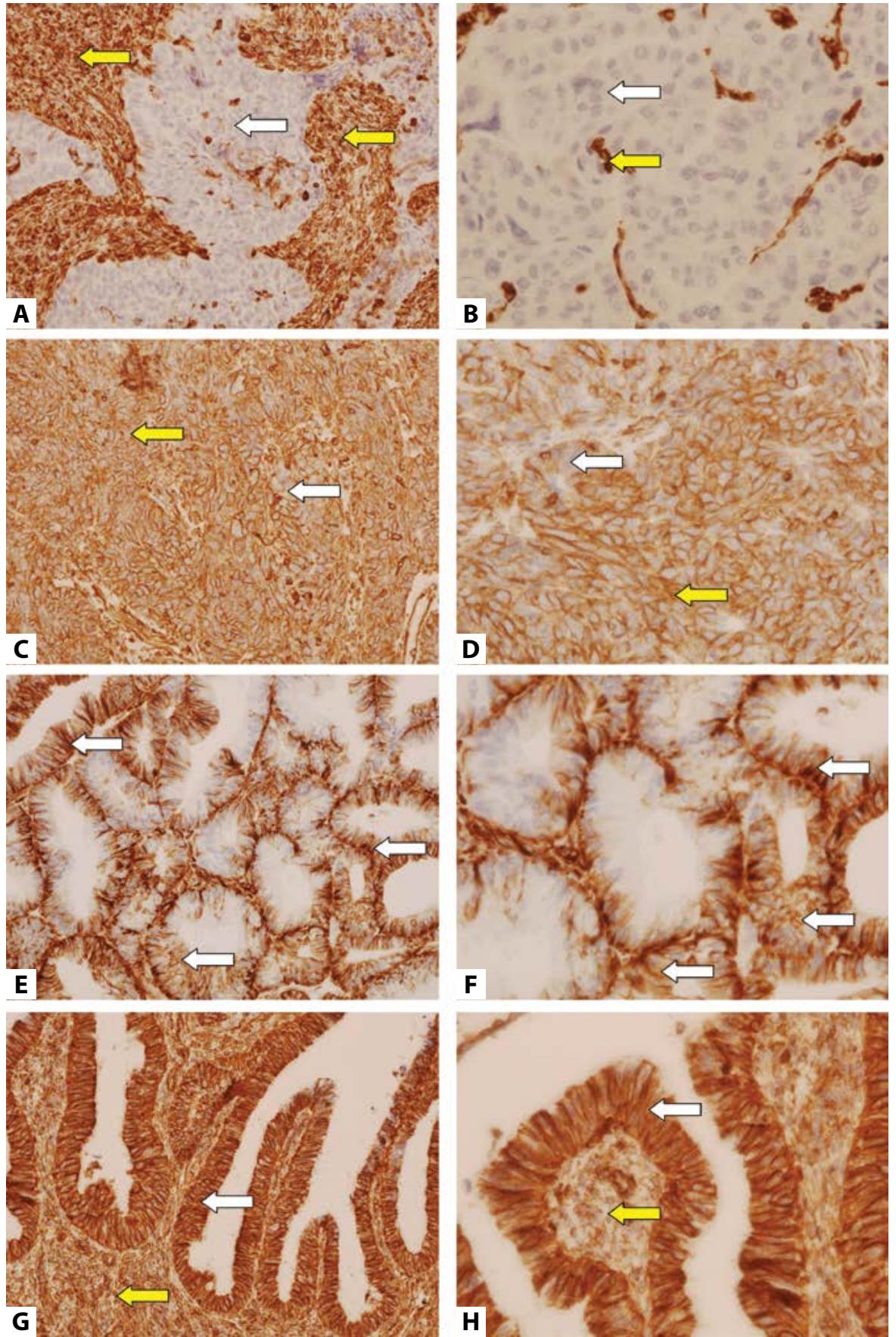

Figure 1. Representative pictures of vimentin expression in analyzed ovarian malignancies. A) and B) Serous adenocarcinoma of the ovary showing absent vimentin expression in cancer cells (white arrows). Vimentin expression is present in the tumor stroma (yellow arrows). Magnification: x200 and x400 for A) and B) respectively; C) and D) Serous adenocarcinoma of the ovary presenting with minimal vimentin expression in cancer cells (white arrows). Vimentin expression is present in the tumor stroma (yellow arrows). Magnification: $\times 200$ and $\times 400$ for C) and D) E) and F) Endometrioid adenocarcinoma of the ovary showing moderate vimentin expression in cancer cells (white arrows). Magnification: x200 and x400 for $\mathbf{E}$ ) and F) respectively; G) and $\mathbf{H}$ ) Serous adenocarcinoma of the ovary with high vimentin expression in cancer cells (white arrows). Vimentin expression is also found in the tumor stroma (yellow arrows). Magnification: $x 200$ and $\times 400$ for $\mathbf{G}$ ) and $\mathbf{H}$ ) respectively

\section{Vimentin expression and clinicopathologic features of ovarian malignancies}

Vimentin expression (in both cancer cells and tumor stroma) did not differ between the histopathological types of ovarian malignancies (Tab. 5). Similarly, there were no differences in vimentin expression with regard to the FIGO stage of the disease and tumor grade (Tab.6). Non-differentiated (2 tumors), transitional cell carcinomas (3 tumors), borderline tumors ( 2 tumors) and G1 neoplasms were all excluded from the analysis of clinicopathologic features due to the low number of subjects. 


\begin{tabular}{|c|c|}
\hline Vimentin expression intensity in cancer cells & $\mathbf{N}$ [\%] \\
\hline Low & $65(69 \%)$ \\
\hline High & 29 (31\%) \\
\hline Vimentin quantitative expression in cancer cells & $\mathbf{N}$ [\%] \\
\hline$<30 \%$ & $16(17.0 \%)$ \\
\hline$\geq 30 \%$ & $78(83.0 \%)$ \\
\hline Vimentin expression intensity in stroma & $\mathbf{N}[\%]$ \\
\hline Low & $14(15 \%)$ \\
\hline High & $80(85 \%)$ \\
\hline Stromal cellularity & $\mathbf{N}$ [\%] \\
\hline Low & $35(37.6 \%)$ \\
\hline High & $58(62.4 \%)$ \\
\hline
\end{tabular}

\section{DISCUSSION}

Numerous studies have revealed high vimentin expression as a negative prognostic factor in human neoplasms. In the case of colorectal cancer, it was shown that both tumor cell expression and tumor stroma expression were associated with poor patient prognosis [19, 20,23]. In a meta-analysis by Du et al., vimentin expression indicated a shortened OS and disease-free survival (DFS), lymph node metastases, and a higher TNM stage of colorectal cancer patients [23]. Similarly, a meta-analysis by Yin et al., of 1.598 patients with gastric cancer,has shown vimentin expression to be a prognostic factor of shortened survival [24]. Meta-analysis studies evaluating the expression of vimentin in non-small cell lung cancers have also shown the negative predictive value of vimentin expression. Similarly, vimentin expression was associated with poor prognoses for patients with breast cancer, cervical cancer, lung adenocarcinomas, oral cancers, bladder cancer, and soft tissue leiomyosarcomas [23, 24]. However, not all studies are in full agreement with these reports of the negative prognostic role of vimentin in cancer patients. In a study by Zou et al. involving more than 500 patients with early stage endometrioid cancers, vimentin expression was associated with prolonged survival [25].

There is sparse data on vimentin tumor expression as a prognostic factor in cases of ovarian cancer. We have identified only one study, by Li et al., which includes analysis of 43 cases of EOC. In their univariate analysis, the authors associate tumor vimentin expression with significantly shortened disease-free survival, whereas in their multivariate analysis the association between vimentin expression and disease-free survival was insignificant. Vimentin expression did not influence EOC patients' OS, in the univariate or multivariate analyses [26]. In our study, we found that both high quantitative vimentin expression and a high intensity of vimentin expression in cancer cells were associated with a better prognosis. This may be explained by the role of vimentin in the regulation of cancer cell platinum-resistance, as this is one of the most important factors influencing patient prognosis [27]. In their study, Huo et al. used two pairs of ovarian cancer cell lines [21]. The authors revealed that the downregulation of vimentin expression increased resistance to cisplatin. Furthermore, their study showed that the upregulation of vimentin expression markedly increased cisplatin sensitivity. The study by Huo et al. is not the only report showing downregulation of vimentin as an indicator of drug resistance in patients with ovarian cancer. Similar results were obtained by Jin et al. where downregulation of vimentin was found among cisplatin-resistant EOC cells [22]. Additionally, Kanakkanthara et al. have shown that low vimentin expression is associated with resistance to microtubule stabilizing agents, such as peloruside $A$ and laulimalide [28]. Taken together, these data suggest that platinum sensitivity related to the expression of vimentin in cancer cells is responsible for the prolonged survival of ovarian cancer patients.

Interestingly, Huo et et al. have revealed that vimentin knockdown cells formed spheroids and they destabilized both anchoring junctions and tight junctions. Additionally, vimentin-downregulated cells acquired stem cell-like characteristics and showed increased expression of stem cell markers [21]. There is a close relationship between cancer stem cell (CSC) phenotype sand cells undergoing EMT, and both are associated with chemoresistance [16]. These findings may indicate that despite acquiring EMT/CSCs features, ovarian cancer cells with higher vimentin expression are characterized by improved sensitivity to platinum-based agents. Thus, an association between vimentin downregulation, EMT, cancer stem-cells phenotypes, and chemoresistance is particularly intriguing and demands further investigation.

We also found that patients with high vimentin expression in the stroma of malignant ovarian tumors had shortened survival compared with patients with low vimentin expression, however, this difference did not reach the level of statistical significance. To the best of our knowledge, our study is the first to report vimentin expression in the stroma of the ovarian neoplasm. We did identify studies showing that a poorer prognosis is related to stromal vimentin expression; but those studies concerned patients with colorectal and prostate cancers $[20,29]$. The tumor stroma mainly consists of resident fibroblasts, endothelial cells, pericytes, leukocytes, and extra-cellular matrix. Within the tumor stroma, the CAFs are the most prominent cell type. Vimentin is one of the markers used toidentify CAFs. However, vimentin staining was also noted in normal fibroblasts present in the tumor stroma and in the tumor stroma myofibroblasts (activated fibroblasts) [13]. In addition, vimentin staining was present on the cells of 

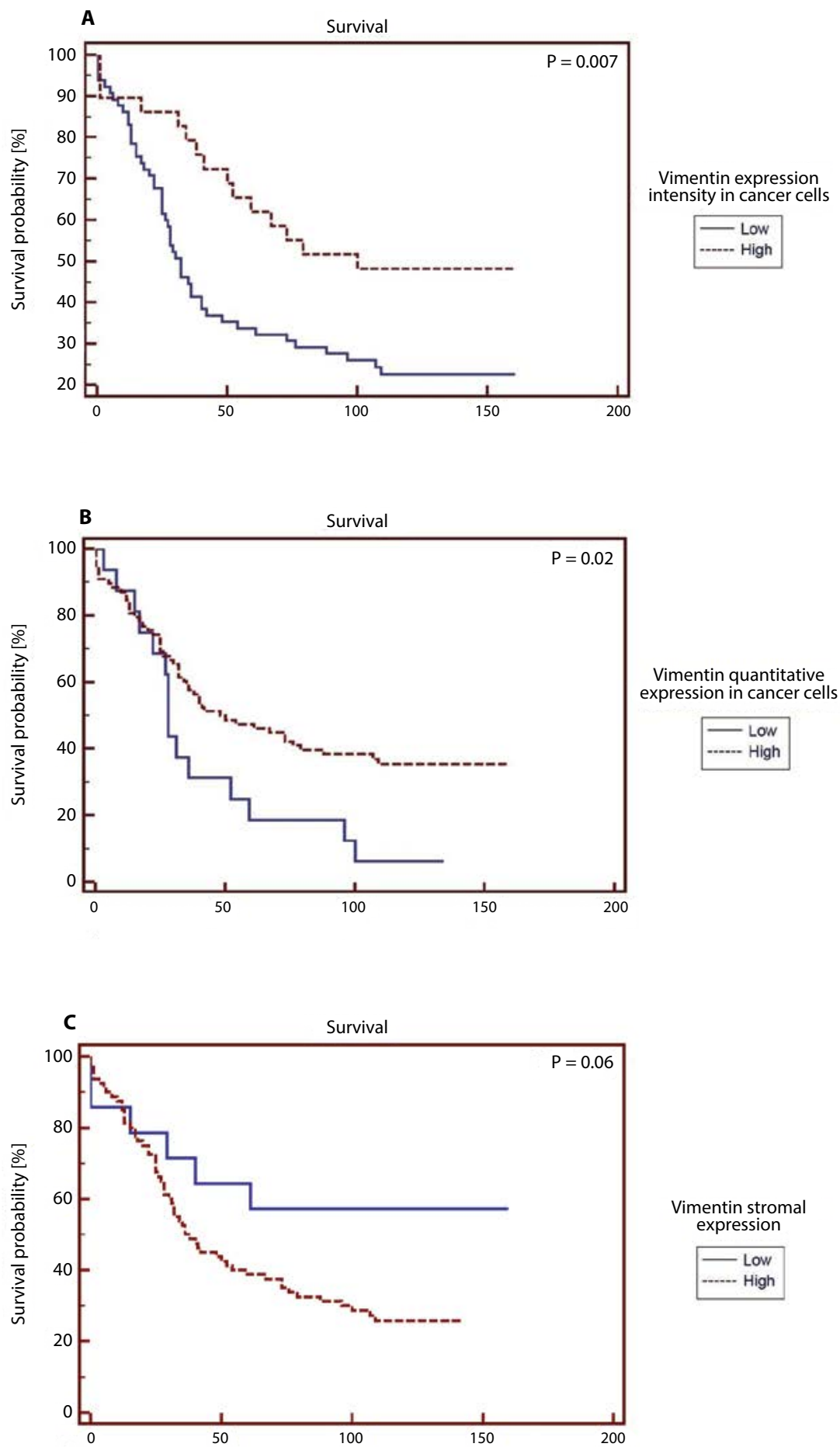

Figure 2. Survival analyses according to vimentin expression. A) Patients with high vs low vimentin expression intensity in cancer cells; B) Patients with high (up to and including $30 \%$ of cells) vs low (below $30 \%$ of cells) vimentin quantitative expression in cancer cells. C) Patients with low and high vimentin expression in the tumor stroma

vascular origin, such as the endothelial cells co-labeled with CD31 and pericytes [14]. Further, vimentin may be secreted into extracellular space by activated macrophages [15].Thus, there are numerous putative sources of tumor stroma vimentin expression. Multiple studies support the role of CAFs in ovarian cancer development and progression; however, the role of normal fibroblasts and myofibroblasts is not fully elucidated [13].Additionally, current studies indicates that there are various subpopulations of CAFs, and that there are significant differences in their impacts on tumor growth [30]. 
Table 3. The relationship between vimentin expression in cancer cells with vimentin stromal expression

\begin{tabular}{|c|c|c|c|}
\hline & $\begin{array}{l}\text { Low vimentin expression intensity } \\
\text { in cancer cells }\end{array}$ & $\begin{array}{l}\text { High vimentin expression } \\
\text { intensity in cancer cells }\end{array}$ & \\
\hline Low vimentin expression intensity in stroma & $14(15 \%)$ & $0(0 \%)$ & \multirow{2}{*}{$P=0.0006$} \\
\hline \multirow[t]{2}{*}{ High vimentin expression intensity in stroma } & $37(39 \%)$ & $43(46 \%)$ & \\
\hline & $\begin{array}{l}\text { Low vimentin expression in } \\
\text { cancer cells ( }<30 \% \text { of cells), N [\%] }\end{array}$ & $\begin{array}{l}\text { High vimentin expression in } \\
\text { cancer cells ( } \geq 30 \% \text { of cells), N [\%] }\end{array}$ & \\
\hline Low vimentin expression intensity in stroma & $5(5 \%)$ & $9(10 \%)$ & \multirow{2}{*}{$P=0.52$} \\
\hline High vimentin expression intensity in stroma & $21(22 \%)$ & $59(63 \%)$ & \\
\hline
\end{tabular}

\begin{tabular}{|c|c|c|c|}
\hline & Low vimentin expression intensity in cancer cells & High vimentin expression intensity in cancer cells & P-value \\
\hline Low stromal cellularity & $21(22 \%)$ & $14(15 \%)$ & \multirow{2}{*}{$P=0.16$} \\
\hline \multirow[t]{2}{*}{ High stromal cellularity } & $44(47 \%)$ & $15(16 \%)$ & \\
\hline & $\begin{array}{l}\text { Low vimentin expression in cancer cells } \\
(<30 \% \text { of cells), } N \text { [\%] }\end{array}$ & $\begin{array}{l}\text { High vimentin expression in cancer cells } \\
\text { ( } \geq 30 \% \text { of cells), } \mathbf{N} \%]\end{array}$ & \\
\hline Low stromal cellularity & $13(14 \%)$ & $22(23 \%)$ & \multirow{2}{*}{$P=0.15$} \\
\hline \multirow[t]{2}{*}{ High stromal cellularity } & $13(14 \%)$ & $46(49 \%)$ & \\
\hline & $\begin{array}{l}\text { Low vimentin expression intensity in tumor } \\
\text { stroma, } \mathrm{N}[\%]\end{array}$ & $\begin{array}{l}\text { High vimentin expression intensity in tumor } \\
\text { stroma, } \mathrm{N}[\%]\end{array}$ & \\
\hline Low stromal cellularity & $11(11 \%)$ & $24(26 \%)$ & \multirow{2}{*}{$P=0.0015$} \\
\hline High stromal cellularity & $3(3 \%)$ & $56(60 \%)$ & \\
\hline
\end{tabular}

Table 5. The relationships between vimentin expression and histopathological types of the disease

\begin{tabular}{|c|c|c|c|c|c|c|}
\hline $\begin{array}{l}\text { Vimentin expression } \\
\text { intensity in the tumor cells }\end{array}$ & $\begin{array}{l}\text { Serous } \\
\text { adenocarcinoma }\end{array}$ & $\begin{array}{l}\text { Mucinous } \\
\text { adenocarcinoma }\end{array}$ & $\begin{array}{l}\text { Clear-cell } \\
\text { adenocarcinoma }\end{array}$ & $\begin{array}{l}\text { Endometrioid } \\
\text { adenocarcinoma }\end{array}$ & $\begin{array}{l}\text { Adult granulosa } \\
\text { cell tumors }\end{array}$ & P-value \\
\hline Low & $37(66 \%)$ & $5(100 \%)$ & $3(75 \%)$ & $6(55 \%)$ & $7(70 \%)$ & \multirow{2}{*}{$P=0.48$} \\
\hline High & $19(34 \%)$ & $0(0 \%)$ & $1(25 \%)$ & $5(45 \%)$ & $3(30 \%)$ & \\
\hline $\begin{array}{l}\text { Vimentin expression in } \\
\text { cancer cells (percentage of } \\
\text { cells) }\end{array}$ & $\begin{array}{l}\text { Serous } \\
\text { adenocarcinoma }\end{array}$ & $\begin{array}{l}\text { Mucinous } \\
\text { adenocarcinoma }\end{array}$ & $\begin{array}{l}\text { Clear-cell } \\
\text { adenocarcinoma }\end{array}$ & $\begin{array}{l}\text { Endometrioid } \\
\text { adenocarcinoma }\end{array}$ & $\begin{array}{l}\text { Adult granulosa } \\
\text { cell tumors }\end{array}$ & \\
\hline Low ( $<30 \%$ of cells), $\mathrm{N}$ [\%] & $11(20 \%)$ & $1(20 \%)$ & $0(0 \%)$ & $2(18 \%)$ & $1(10 \%)$ & \multirow{2}{*}{$P=0.84$} \\
\hline High ( $\geq 30 \%$ of cells), N [\%] & $45(80 \%)$ & $4(80 \%)$ & $4(100 \%)$ & $9(82 \%)$ & $9(90 \%)$ & \\
\hline $\begin{array}{l}\text { Vimentin expression } \\
\text { intensity in tumor stroma }\end{array}$ & $\begin{array}{l}\text { Serous } \\
\text { adenocarcinoma }\end{array}$ & $\begin{array}{l}\text { Mucinous } \\
\text { adenocarcinoma }\end{array}$ & $\begin{array}{l}\text { Clear-cell } \\
\text { adenocarcinoma }\end{array}$ & $\begin{array}{l}\text { Endometrioid } \\
\text { adenocarcinoma }\end{array}$ & $\begin{array}{l}\text { Adult granulosa } \\
\text { cell tumors }\end{array}$ & \\
\hline Low & $7(8 \%)$ & $0(0 \%)$ & $0(0 \%)$ & $1(1 \%)$ & $4(5 \%)$ & \multirow{2}{*}{$P=0.11$} \\
\hline High & $50(57 \%)$ & $5(6 \%)$ & $4(5 \%)$ & $10(12 \%)$ & $6(7 \%)$ & \\
\hline
\end{tabular}

Although high tumor microvascular density (MVD) is known as apoor prognostic indicator in many human neoplasms, in general, MVD assessed by endothelial staining with an anti-CD31 antibody does not influence ovarian cancer patient prognosis [31]. High vimentin expression may also reflect increased pericyte coverage of tumor blood vessels [14]. On the one hand, tumor vessels with high pericyte coverage build up a more stable vasculature leading to increased tumor growth. On the other hand, high pericyte coverage inhibits tumor hematogenous metastases and is associated with better prognoses for cancer patients [32]. Recent findings suggest that the spread of ovarian cancer by the hematogenous route in patients is far more significant than previously thought [33].Taken together, these findings show a trend towards a poor prognosis for patients with tumors presenting with high vimentin stromal expression. However, considering the variety of vimentin positive cells within the tumor stroma, further studies are needed for a better identification of these cells.

Recently, attention has been paid to interactions between cancer cells and the tumor stroma structure. In this paper, we have evaluated the relationships between vimentin expression in cancer cells with vimentin stromal expression and tumor stroma cellularity. The idea behind this 
Table 6. Vimentin expression and clinicopathological features of the disease

\begin{tabular}{|c|c|c|c|}
\hline & Low vimentin expression intensity in cancer cells & High vimentin expression intensity in cancer cells & P-value \\
\hline FIGO I and II & $20(21 \%)$ & $8(9 \%)$ & \multirow{2}{*}{$P=0.94$} \\
\hline FIGO III & $45(48 \%)$ & $21(22 \%)$ & \\
\hline G2 & $19(23 \%)$ & $11(13 \%)$ & \multirow{2}{*}{$P=0.50$} \\
\hline \multirow[t]{2}{*}{ G3 } & $38(45 \%)$ & $14(19 \%)$ & \\
\hline & $\begin{array}{l}\text { Low vimentin expression in cancer cells } \\
(<30 \% \text { of cells), } \mathbf{N}[\%]\end{array}$ & $\begin{array}{l}\text { High vimentin expression in cancer cells } \\
\text { ( } \geq 30 \% \text { of cells), } N[\%]\end{array}$ & \\
\hline FIGO I and II & $7(7 \%)$ & $21(22 \%)$ & \multirow{2}{*}{$P=0.90$} \\
\hline FIGO III & $19(20 \%)$ & $47(51 \%)$ & \\
\hline G2 & 9 & 21 & \multirow{2}{*}{$P=0.81$} \\
\hline \multirow[t]{2}{*}{ G3 } & 13 & 39 & \\
\hline & Low vimentin expression intensity in tumor stroma, $\mathrm{N}$ [\%] & High vimentin expression intensity in tumor stroma, $\mathrm{N}$ [\%] & \\
\hline FIGO I and II & $6(6 \%)$ & $22(23 \%)$ & \multirow{2}{*}{$P=0.40$} \\
\hline FIGO III & $8(9 \%)$ & $58(62 \%)$ & \\
\hline G2 & $3(5 \%)$ & $27(32 \%)$ & \multirow{2}{*}{$P=0.96$} \\
\hline G3 & $4(6 \%)$ & 48 (57\%) & \\
\hline
\end{tabular}

project was to investigate vimentin as an indicator of stroma remodeling. We have shown that cancer cell vimentin expression strongly correlates with a degree of tumor stroma vimentin expression. Vimentin may be upregulated by a variety of factors, including cancer-related growth factors, such as the transforming growth factor beta (TGF-beta) and the epidermal growth factor (EGF) [34]. Thus, vimentin stromal expression may merely reflect tumor-stroma interactions, whereas cancer cells may influence the stroma compartment. However, vimentin stromal expression may also be explained as a result of specific tumor microenvironmental conditions. For instance, it has been shown that tumor hypoxia through hypoxia-inducible factor-1 (HIF-1) is one of the key elements upregulating the expression of vimentin [35]. In our observations, vimentin expression in tumor stroma was correlated with stroma cellularity. This suggests that vimentin is responsible for, or is just an indicator of, higher stroma infiltration by CAFs and leucocytes. Although we did not observe a correlation between stroma cellularity and the patients' prognoses, EOC with well-developed stroma are characterized by poor prognoses [4]. However, recent studies highlight that the stroma content differs significantly between various cases of EOCs. Additionally, there are various subpopulations of fibroblasts infiltrating tumor stroma. For instance, Givel et al., have shown that the CAF-S1 subset, when present in the stroma of high grade serous ovarian carcinoma has more potent immunosuppressive characteristics when compared with other subsets of CAFs. Additionally, there are various populations of TILs, and while regulatory $\mathrm{T}$-cells indicate poor survival, cytotoxic T-cells indicate better prognoses for cancer patients [9]. These findings suggest that the prognosis of cancer patients is more related to the type of stroma infiltrating cells, and less to their number [30].

\section{CONCLUSIONS}

We conclude that vimentin expression in cancer cells may be used as a potential prognostic factor of the overall survival of patients with ovarian malignancies. Additionally, due to the interactions between vimentin expression in cancer cells with vimentin stromal expressions and stroma cellularity, we suggest vimentin may participate in the formation of tumor stroma.

\section{Acknowledgments}

We would like to thank Christine Maisto for her assistance with the manuscript.

\section{Financial disclosure}

All laboratory studies were financed by the Department of Tumour Pathology and Pathomorphology, the Ludwik Rydygier Collegium Medicum in Bydgoszcz, Nicolaus Copernicus University in Torun, Poland

\section{REFERENCES}

1. Szymankiewicz M, Dziobek K, Sznajdorwska M, et al. An analysis of the influence of infection on overall survival rates, following modified posterior pelvic exenteration for advanced ovarian cancer. Ginekol Pol. 2018; 89(11): 618-626, doi: 10.5603/GP.a2018.0106, indexed in Pubmed: 30508214

2. Szymankiewicz M, Koper K, Dziobek K, et al. Microbiological monitoring in patients with advanced ovarian cancer before and after cytoreductive surgery - a preliminary report. Current Issues in Pharmacy and Medical Sciences. 2017; 30(4): 198-202, doi: 10.1515/cipms-2017-0038.

3. Baldwin LA, Huang B, Miller RW, et al. Ten-year relative survival for epithelial ovarian cancer. Obstet Gynecol. 2012; 120(3): 612-618, doi: 10.1097/AOG.0b013e318264f794, indexed in Pubmed: 22914471. 
4. Chen Y, Zhang L, Liu W, et al. Prognostic Significance of the Tumor-Stroma Ratio in Epithelial Ovarian Cancer. Biomed Res Int. 2015; 2015: 589301, doi: 10.1155/2015/589301, indexed in Pubmed: 26609529.

5. Bremnes RM, Dønnem T, Al-Saad S, et al. The role of tumor stroma in cancer progression and prognosis: emphasis on carcinoma-associated fibroblasts and non-small cell lung cancer. JThorac Oncol. 2011;6(1): 209-217, doi: 10.1097/JTO.0b013e3181f8a1bd, indexed in Pubmed: 21107292.

6. Naito $Y$, Yoshioka $Y$, Yamamoto $Y$, et al. How cancer cells dictate their microenvironment: present roles of extracellular vesicles. Cell Mol Life Sci. 2017; 74(4): 697-713, doi: 10.1007/s00018-016-2346-3, indexed in Pubmed: 27582126.

7. Szubert $S$, Moszynski R, MichalakS, et al. The associations between serum VEGF, bFGF and endoglin levels with microvessel density and expression of proangiogenic factors in malignant and benign ovarian tumors. Microvasc Res. 2016; 107: 91-96, doi: 10.1016/j.mvr.2016.06.002, indexed in Pubmed: 27312585.

8. Walentowicz-Sadlecka M, Dziobek K, Grabiec M, et al. The analysis of human leukocyte antigen-G level in patients with endometrial cancer by Western blot technique. Am J Reprod Immunol. 2018 [Epub ahead of print], doi: 10.1111/aji.13070, indexed in Pubmed: 30414280.

9. Biedka M, Nowikiewicz T, Dziobek K, et al. The analysis of Treg lymphocytes in the blood of patients with breast cancer during the combined oncological treatment. Breast J. 2019;26(1).

10. Kakarla S, Song XT, Gottschalk S. Cancer-associated fibroblasts as targets for immunotherapy. Immunotherapy. 2012; 4(11): 1129-1138, doi: 10.2217/imt.12.112, indexed in Pubmed: 23194363.

11. Satelli A, Li S. Vimentin in cancer and its potential as a molecular target for cancer therapy. Cell Mol Life Sci. 2011; 68(18): 3033-3046, doi: 10.1007/s00018-011-0735-1, indexed in Pubmed: 21637948.

12. Sommers $\mathrm{CL}$, Walker-Jones $\mathrm{D}$, Heckford $\mathrm{SE}$, et al. Vimentin rather than keratin expression in some hormone-independent breast cancer cell lines and in oncogene-transformed mammary epithelial cells. Cancer Res. 1989; 49(15): 4258-4263, indexed in Pubmed: 2472876.

13. Wang $B, X i C$, Liu M, et al. Breast fibroblasts in both cancer and normal tissues induce phenotypic transformation of breast cancer stem cells: a preliminary study. PeerJ. 2018; 6: e4805, doi: 10.7717/peerj.4805, indexed in Pubmed: 29780673.

14. Díaz-Flores $\mathrm{L}$, Gutiérrez $\mathrm{R}$, Varela $\mathrm{H}$, et al. Microvascular pericytes: a review of their morphological and functional characteristics. Histol Histopathol. 1991; 6(2): 269-286, indexed in Pubmed: 1802127.

15. Mor-Vaknin N, Punturieri A, Sitwala K, et al. Vimentin is secreted by activated macrophages. Nat Cell Biol. 2003; 5(1): 59-63, doi: 10.1038/ncb898, indexed in Pubmed: 12483219.

16. Sato R, Semba T, Saya H, et al. Concise Review: Stem Cells and Epithelial-Mesenchymal Transition in Cancer: Biological Implications and Therapeutic Targets. Stem Cells. 2016; 34(8): 1997-2007, doi: 10.1002/stem.2406, indexed in Pubmed: 27251010.

17. Kidd ME, Shumaker DK, Ridge KM. The role of vimentin intermediate filaments in the progression of lung cancer. Am J Respir Cell Mol Biol. 2014; 50(1): 1-6, doi: 10.1165/rcmb.2013-0314TR, indexed in Pubmed: 23980547.

18. Bauer A, Mylroie $H$, Thornton CC, et al. Identification of cyclins A1, E1 and vimentin as downstream targets of heme oxygenase- 1 in vascular endothelial growth factor-mediated angiogenesis. Sci Rep. 2016; 6: 29417, doi: 10.1038/srep29417, indexed in Pubmed: 27388959.

19. Ngan $\mathrm{CY}$, Yamamoto $\mathrm{H}$, Seshimo l, et al. Quantitative evaluation of vimentin expression in tumour stroma of colorectal cancer. Br J Cancer. 2007; 96(6): 986-992, doi: 10.1038/sj.bjc.6603651, indexed in Pubmed: 17325702.

20. Liu LG, Yan XB, Xie RT, et al. Stromal Expression of Vimentin Predicts the Clinical Outcome of Stage II Colorectal Cancer for High-Risk Patients. Med Sci Monit. 2017; 23: 2897-2905, indexed in Pubmed: 28611349.
21. Huo Yi, Zheng Z, Chen Y, et al. Downregulation of vimentin expression increased drug resistance in ovarian cancer cells. Oncotarget. 2016; 7(29): 45876-45888, doi: 10.18632/oncotarget.9970, indexed in Pubmed: 27322682.

22. Jin L, Huo Yi, Zheng Z, et al. Down-regulation of Ras-related protein Rab 5C-dependent endocytosis and glycolysis in cisplatin-resistant ovarian cancer cell lines. Mol Cell Proteomics. 2014; 13(11): 3138-3151, doi: 10.1074/mcp.M113.033217, indexed in Pubmed: 25096996.

23. Du Le, Li J, Lei L, et al. High Vimentin Expression Predicts a Poor Prognosis and Progression in Colorectal Cancer: A Study with Meta-Analysis and TCGA Database. Biomed Res Int. 2018; 2018: 6387810, doi: 10.1155/2018/6387810, indexed in Pubmed: 29955607.

24. Yin S, Chen FF, Yang GF. Vimentin immunohistochemical expression as a prognostic factor in gastric cancer: A meta-analysis. Pathol Res Pract. 2018; 214(9): 1376-1380, doi: 10.1016/j.prp.2018.07.014, indexed in Pubmed: 30078472.

25. Zou S, Sun H, Fan L, et al. Prognostic indicators in patients with early stage endometrioid adenocarcinoma: a retrospective case-control study of 523 patients. Int J Clin Exp Med. 2017; 10: 3692-8.

26. Li X, Yang J, Wang X, et al. Role of TWIST2, E-cadherin and Vimentin in epithelial ovarian carcinogenesis and prognosis and their interaction in cancer progression. Eur J Gynaecol Oncol. 2016; 37(1): 100-108, indexed in Pubmed: 27048119.

27. Slaughter K, Holman LL, Thomas EL, et al. Primary and acquired platinum-resistance among women with high grade serous ovarian cancer. Gynecol Oncol. 2016; 142(2): 225-230, doi: 10.1016/j.ygyno.2016.05.020, indexed in Pubmed: 27208536.

28. Kanakkanthara A, Rawson P, Northcote PT, et al. Acquired resistance to peloruside $A$ and laulimalide is associated with downregulation of vimentin in human ovarian carcinoma cells. Pharm Res. 2012; 29(11): 3022-3032, doi: 10.1007/s11095-012-0773-x, indexed in Pubmed: 22584948.

29. Wu JP, Huang WB, Zhou H, et al. Intensity of stromal changes is associated with tumor relapse in clinically advanced prostate cancer after castration therapy. Asian J Androl. 2014; 16(5): 710-714, doi: 10.4103/1008682X.129131, indexed in Pubmed: 24875819.

30. Givel AM, Kieffer Y, Scholer-Dahirel A, et al. miR200-regulated CXCL12 $\beta$ promotes fibroblast heterogeneity and immunosuppression in ovarian cancers. Nat Commun. 2018; 9(1): 1056, doi: 10.1038/s41467-01803348-z, indexed in Pubmed: 29535360.

31. Szubert S, Szpurek D, Moszynski R, et al. Extracellular matrix metalloproteinase inducer (EMMPRIN) expression correlates positively with active angiogenesis and negatively with basic fibroblast growth factor expression in epithelial ovarian cancer. J Cancer Res Clin Oncol. 2014; 140(3): 361-369, doi: 10.1007/s00432-013-1569-z, indexed in Pubmed: 24374756.

32. Cooke VG, LeBleu VS, Keskin D, et al. Pericyte depletion results in hypoxia-associated epithelial-to-mesenchymal transition and metastasis mediated by met signaling pathway. Cancer Cell. 2012; 21(1): 66-81, doi: 10.1016/j.ccr.2011.11.024, indexed in Pubmed: 22264789.

33. Yeung TL, Leung CS, Yip KP, et al. Cellular and molecular processes in ovarian cancer metastasis. A Review in the Theme: Cell and Molecular Processes in Cancer Metastasis. Am J Physiol Cell Physiol. 2015; 309(7): C444-C456, doi: 10.1152/ajpcell.00188.2015, indexed in Pubmed: 26224579.

34. Yates B, Zetterberg C, Rajeev V, et al. Promoter-independent regulation of vimentin expression in mammary epithelial cells by val(12)ras and TGFbeta. Exp Cell Res. 2007; 313(17): 3718-3728, doi: 10.1016/j. yexcr.2007.07.026, indexed in Pubmed: 17719575.

35. Krishnamachary B, Berg-Dixon S, Kelly B, et al. Regulation of colon carcinoma cell invasion by hypoxia-inducible factor 1. Cancer Res. 2003; 63(5): 1138-1143, indexed in Pubmed: 12615733. 